Ciência e Natura, Santa Maria, v. 37, n.4, set-dez 2015, p. 625-633

Revista do Centro de Ciências Naturais e Exatas - UFSM

ISSN impressa: 0100-8307 ISSN on-line: 2179-460X

\title{
Avaliação de metais nos peixes Astyanax altiparanae, Leporinus friderici e Hypostomus strigaticeps, coletados no córrego Curral de Arame, Dourados, MS
}

\author{
Heavy metals in the fishes Astyanax altiparanae, Leporinus friderici and Hypostomus strigaticeps \\ collected in the stream Curral de Arame, Dourados, MS
}

Giovana Tôrres Rossoํ, Cynthia de Barros Mansur¹, Yzel Rondon Súarez², Silvia Cristina Heredia-

\author{
Vieira $^{1}$, Claudia Andrea Lima Cardoso ${ }^{1}$
}

${ }^{1}$ Programa de Pós-Graduação em Recursos Naturais, Centro de Pesquisa em Biodiversidade (CP-Bio), UEMS,

Dourados-MS, Brasil

${ }^{2}$ Programa de Pós-Graduação em Recursos Naturais, Centro Integrado de Análise e Monitoramento Ambiental (CINAM), UEMS, Dourados-MS, Brasil

\section{Resumo}

O lançamento de metais no ambiente causa uma série de problemas ambientais. Portanto, este estudo tem como objetivo avaliar a concentração dos metais ferro, zinco, cobre, níquel e cromo em Astyanax altiparanae, Leporinus friderici e Hypostomus strigaticeps coletados no córrego Curral de Arame, Dourados, MS. As coletas de peixes foram realizadas durante oito meses em quatro pontos do córrego. As análises de metais foram realizadas empregando absorção atômica. $O$ zinco evidenciou melhor a diferença entre os pontos de coleta. O peixe de fundo apresentou maior concentração de metais em relação às outras espécies. Os resultados apresentados indicam que as amostras de peixes não excederam as concentraçães de metais permitidas pela legislação brasileira, mostrando-se que os peixes avaliados são boas fontes dos metais analisados, considerando os valores de ingestão diária recomendada.

Palavras-chave: Cobre, zinco, cromo, ferro, níquel.

\section{Abstract}

The release of metals in the environment causes a series of environmental problems. Therefore, this study aims to evaluate the concentration of the metals iron, zinc, copper, nickel and chromium Astyanax altiparanae, Leporinus friderici $e$ Hypostomus strigaticeps collected in the stream Curral de Arame, Dourados, MS. The fish samples were collected during eight months in four points of the stream. The metal analyzes were performed using atomic absorption. Zinc better showing the difference between the collection points. The bottom fish had a higher concentration of metals in relation to other species. The results presented indicate that the fish samples did not exceed the metal concentrations allowed by Brazilian law, showing that the assessed fish are good sources of the metals analyzed, considering the values of Recommended Daily Intake.

Keywords: Copper, zinc, chromium, iron, nickel. 


\section{Introdução}

$\mathrm{O}$ s metais são componentes naturais de ambientes aquáticos, porém, seus níveis estão aumentando já que estes têm sido lançados no meio ambiente através da poluição de veículos, devido às atividades industriais, por combustíveis fósseis, além de atividades agrícolas e de mineração. A presença de metais no ambiente causa uma série de problemas ambientais e torna-se uma ameaça à saúde dos organismos ali presentes e, indiretamente, dos seres humanos (Kalay e Canli, 2000; Gorur et al., 2012).

As concentrações naturais dos metais no meio estão, geralmente, na ordem de parte por milhão ou parte por bilhão, que representa quantidade necessária para a manutenção dos ciclos químicos, e a preocupação está em ambientes onde estas concentrações passam do limite natural e se tornam tóxicas (Lemes, 2001). Estes metais podem bioacumular e, uma vez acumulados nos tecidos dos animais do meio em que se encontram, podem atingir níveis elevados, causando riscos aos animais e ao meio ambiente (Vandecasteele e Block, 1991).

Os seres humanos são expostos a uma grande gama de metais, embora muitos destes estejam presentes naturalmente no meio (Dural et al., 2007). Alguns metais como cromo, níquel, ferro, cobre e zinco são considerados elementos essenciais ao metabolismo humano, mas em excesso podem levar a distúrbios nos organismos e até à morte (Virga et al., 2007). Geralmente, suas concentrações naturais no meio estão na ordem de parte por milhão ou parte por bilhão, que representa quantidade necessária para a manutenção dos ciclos químicos, e a preocupação está em ambientes onde estas concentrações passam do limite natural e se tornam tóxicas (Lemes, 2001).

Diversos produtos químicos, compostos tóxicos ou persistentes no meio, como os metais, são utilizados em todo o mundo (UNEP, 2011), portanto, torna-se imprescindível o estudo da presença de metais no meio ambiente, devido à sua resistência e abundância na crosta terrestre.
Neste contexto, este estudo teve como objetivo avaliar o teor de $\mathrm{Cr}, \mathrm{Fe}, \mathrm{Ni}, \mathrm{Zn}$ e $\mathrm{Cu}$ em três espécies de peixes (Astyanaxaltiparanae, Leporinusfriderici e Hypostomusstrigaticeps) freqüentes no córrego Curral de Arame, localizado no Município de Dourados/MS, em avaliações sazonais e espaciais.

\section{Material e métodos}

\subsection{Coleta das amostras e armazenamento}

A área em estudo localiza-se em Dourados, MS (latitude 22013'18.54" Sul, longitude $54^{\circ} 48^{\prime} 23.09^{\prime \prime}$ Oeste e altitude de $452 \mathrm{~m}$, Figura 1). A região é constituída por fazendas onde predominam as culturas de milho, soja, feijão e, recentemente, cana-de-açúcar. Tem clima tropical com inverno seco e verão úmido, apresentando médias anuais de precipitação entre $1400-1500 \mathrm{~mm}$.

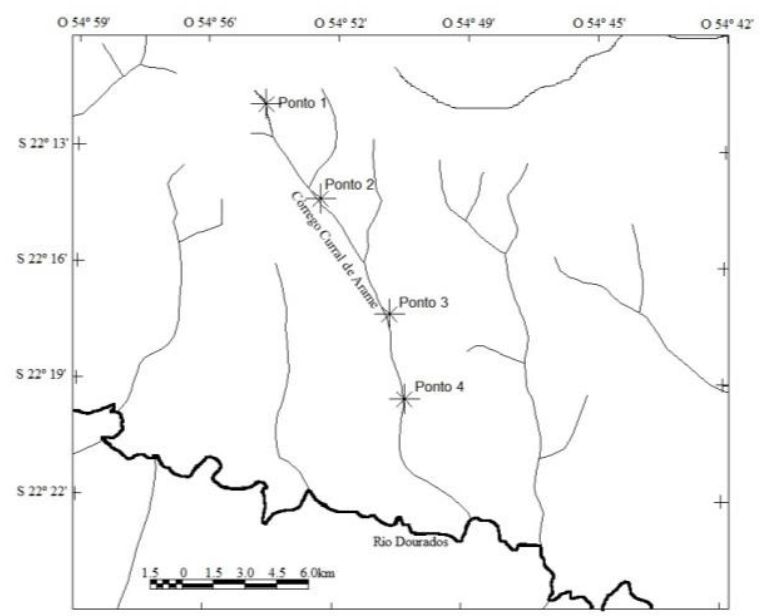

Figura 1 - Mapa da localização dos pontos de coleta ao longo do córrego Curral de Arame, Dourados, MS, vista geral dos pontos.

As coletas foram realizadas nos meses de outubro (Out/11) e dezembro (Dez/11) de 2011 e nos meses de fevereiro (Fev/12), abril (Abril/12) e junho (Jun/12) de 2012, sendo que em Out/11 choveu 127,7 mm, em Dez/11, 77,6 mm, em Fev/12, 122,0 mm, em Abril/12, 187,8 mm e em $\mathrm{Jun} / 12$ choveu 216,8 mm (dados de pluviosidade obtidos da Embrapa Agropecuária Oeste). 
A coleta das amostras foi distribuída em quatro pontos (Figura 1) onde é possível observar uma mescla de vegetação de Cerrado muitas vezes bastante depreciada e plantações das culturas típicas da região.

Em cada local de amostragem foram armadas duas redes de espera com malhas variando entre 1,5 e $4,0 \mathrm{~cm}$ de entrenós adjacentes, que permaneceram no local por $24 \mathrm{~h}$. No ponto 1 não foram armadas redes em nenhuma das coletas por se tratar das proximidades da nascente do rio. Após as $24 \mathrm{~h}$, todos os exemplares de peixes presos nas redes foram coletados e acondicionados em sacos de polietileno, etiquetados e, então, depositados em uma caixa térmica para serem transportados ao laboratório. Os peixes foram armazenados em freezer à $-5^{\circ} \mathrm{C}$, para posterior retirada da musculatura para a realização das análises. Para cada ponto de coleta os exemplares variaram de 5 a 12 para cada espécie.

Entre a coleta e a finalização das análises dos metais, o tempo médio foi de 30 dias para todas as matrizes.

\subsection{Preparo e extração das amostras}

As amostras de peixe foram descongeladas à temperatura ambiente, secas em estufa a $50^{\circ} \mathrm{C}$ por $2 \mathrm{~h}$ e, em seguida, $1 \mathrm{~g}$ da musculatura seca foi transferida para tubos de ensaio de boro silicato. Adicionaram-se $10 \mathrm{~mL}$ de $\mathrm{HNO}_{3}$ concentrado e $2,5 \mathrm{~mL}$ de $\mathrm{HClO}_{4}$ concentrado, deixando reagir por $24 \mathrm{~h}$. A amostra foi levada a um tubo digestor a $600^{\circ} \mathrm{C}$ e aquecida até próximo à secura. Posteriormente, foram resfriadas, redissolvidas em $3 \mathrm{~mL}$ de água ultrapurificada (Human UP 900/Scholar-UV), foi adicionado $1 \mathrm{~mL}$ de $\mathrm{HCl}$ concentrado e, o volume, completado com água ultrapurificada em balão volumétrico de $5 \mathrm{~mL}$. As amostras foram transferidas para frascos de polietileno para a realização das leituras, sendo diluídas quando necessário. As análises foram realizadas seguindo metodologia descrita por Clesceri et al. (1996).

\subsection{Procedimentos analíticos para determinação dos teores de metais}

As leituras foram realizas em triplicata utilizando-se espectrofotômetro de absorção atômica, da marca Shimadzu, modelo AA7000, empregando-se ar/acetileno.

Os metais analisados foram $\mathrm{Cr}, \mathrm{Fe}, \mathrm{Ni}$, $\mathrm{Zn}$ e $\mathrm{Cu}$. As condições experimentais de análises para cada metal estão descritas na Tabela 1.

As concentrações dos metais presentes nas amostras das três matrizes (peixe, água e sedimento), foram calculadas a partir da construção de curvas de calibração específicas para cada elemento, com no mínimo cinco pontos e apresentando coeficientes de determinação iguais ou superiores a 0,9992 (Tabela 1).

Tabela 1 - Condições operacionais utilizadas para as medidas de espectrofotometria de absorção atômica na determinação dos metais estudados e o coeficiente de determinação obtido empregando-se o método de padrão externo.

\begin{tabular}{cccc}
\hline Elemento & $\begin{array}{c}\text { Faixa linear } \\
\left(\mathrm{mg} \mathrm{L}^{-1}\right)\end{array}$ & $\lambda(\mathrm{nm})$ & $\begin{array}{c}\text { Coeficiente de } \\
\text { determinação }\left(\mathrm{R}^{2}\right)\end{array}$ \\
\hline $\mathrm{Cr}$ & $0,1-12,0$ & 357,9 & 0,9998 \\
$\mathrm{Fe}$ & $0,2-9,0$ & 248,3 & 0,9996 \\
$\mathrm{Ni}$ & $0,2-8,0$ & 232,0 & 0,9992 \\
$\mathrm{Zn}$ & $0,1-6,0$ & 213,9 & 0,9992 \\
$\mathrm{Cu}$ & $0,2-6,0$ & 324,7 & 0,9994 \\
\hline
\end{tabular}

\section{Resultados e discussão}

As espécies de peixes avaliadas no estudo foram Astyanax altiparanae (Lambari de calda amarela), Leporinus friderici (Piau) e Hypostomus strigaticeps (Cascudo), estas foram espécies coletadas em maior quantidade e que frequentemente são pescadas na região. Em função do consumo de algumas espécies por pescadores locais, a musculatura foi a parte dos peixes escolhida para ser analisada. A amostragem de peixes apresentou variações ao 
longo das coletas e, este fato, muitas vezes, está ligado à condição climática ou, até mesmo, à instabilidade do córrego, acarretando, assim, em uma carência de exemplares de peixes.

Não foram encontrados, na literatura, estudos que descrevam análises relacionadas a metais em rios e córregos de Mato Grosso do Sul com as espécies avaliadas neste estudo. Quanto à A. altiparanae, Orsi et al. (2004) realizaram um estudo sobre a estrutura populacional no médio rio Paranapanema no Paraná e Gomiero e Braga (2003) estudaram a alimentação desta espécie com enfoque para a ingestão de sementes na bacia do rio Corumbataí em São Paulo.
Em estudo realizado por BeneditoCecilio et al. (2005), foram avaliadas as variações fisiológicas de $L$. friderici em decorrência de alterações ambientais, no reservatório do Corumbá em Goiás. Braga (2001) estudou parâmetros de crescimento e mortalidade desta espécie no reservatório de Volta Grande nos estados de Minas Gerais e São Paulo. Borba et al. (2010), com o intuito de buscar marcadores populacionais, realizou análises filogenéticas e filogeográficas em populações de H. strigaticeps na bacia do Alto Rio Paraná.

Os valores de $\mathrm{pH}$ e condutividade das amostras de água em cada ponto são descritos na Tabela 2.

Tabela 2 - Valores de parâmetros físico-químicos obtidos com amostras de água coletadas no córrego Curral de Arame.

\begin{tabular}{ccccc}
\hline \multicolumn{5}{c}{$\mathrm{pH}(\mathrm{M} \pm \mathrm{DP})$} \\
\hline P1 & $\mathrm{P} 2$ & $\mathrm{P} 3$ & $\mathrm{P} 4$ \\
\hline Out $/ 11$ & $7,64 \pm 0,01$ & $7,54 \pm 0,01$ & $7,43 \pm 0,01$ & $7,35 \pm 0,03$ \\
Fev/11 & $5,69 \pm 0,01$ & $5,98 \pm 0,07$ & $6,66 \pm 0,06$ & $6,60 \pm 0,06$ \\
Abril/12 & $7,22 \pm 0,01$ & $5,95 \pm 0,08$ & $5,11 \pm 0,09$ & $5,13 \pm 0,02$ \\
Jun/12 & $6,30 \pm 0,06$ & $5,75 \pm 0,11$ & $5,60 \pm 0,01$ & $5,42 \pm 0,13$ \\
\hline \multicolumn{5}{c}{ Cond $(\mu \mathrm{HS} / \mathrm{cm})(\mathrm{M} \pm \mathrm{DP})$} \\
\hline Out $/ 11$ & $6,05 \pm 0,02$ & $48,20 \pm 0,46$ & $39,70 \pm 0,15$ & $46,10 \pm 0,12$ \\
Dez/11 & $45,40 \pm 0,05$ & $60,71 \pm 0,01$ & $52,97 \pm 0,05$ & $60,93 \pm 0,01$ \\
Fev/12 & $52,68 \pm 0,01$ & $52,20 \pm 0,06$ & $52,40 \pm 0,09$ & $50,50 \pm 0,01$ \\
Abril/12 & $44,90 \pm 0,05$ & $27,30 \pm 0,18$ & $39,50 \pm 0,03$ & $31,60 \pm 0,08$ \\
Jun/12 & $42,80 \pm 0,03$ & $39,60 \pm 0,05$ & $38,40 \pm 0,02$ & $38,70 \pm 0,01$ \\
\hline
\end{tabular}

pH: potencial hidrogeniônico, Cond.: condutividade, M: média, DP: desvio padrão. Valores de referência do CONAMA: O.D. inferior a 4,0 mg/L; pH entre 6,00-9,00; e não menciona valores de condutividade.

Os valores mais elevados de $\mathrm{pH}$ e influenciaram nas concentrações dos metais em condutividade da água nas primeiras coletas não peixes (Tabela 3). 
Tabela 3 - Valores médios de metais em amostras de peixes do córrego Curral de Arame, Dourados, MS.

\begin{tabular}{|c|c|c|c|c|c|}
\hline \multicolumn{6}{|c|}{ Concentrações médias $(\mathrm{mg} / \mathrm{kg}) \pm$ desvio padrão } \\
\hline & \multicolumn{3}{|c|}{ Astyanax altiparanae } & \multicolumn{2}{|c|}{ Leporinus friderici } \\
\hline $\mathrm{Zn}$ & P2 & P3 & P4 & P2 & P3 \\
\hline${ }^{*}$ Out/11 & NC & $24,60 \pm 0,01$ & $24,30 \pm 0,01$ & $20,10 \pm 0,02$ & $\mathrm{NC}$ \\
\hline${ }^{*}$ Dez/11 & NC & NC & NC & $21,20 \pm 0,01$ & $21,60 \pm 0,01$ \\
\hline${ }^{*} \mathrm{Fev} / 12$ & $24,02 \pm 0,01$ & $24,40 \pm 0,01$ & $24,25 \pm 0,01$ & $21,20 \pm 0,01$ & $21,20 \pm 0,01$ \\
\hline$* * \mathrm{Abr} / 12$ & $25,25 \pm 0,01$ & $\mathrm{NC}$ & $\mathrm{NC}$ & $\mathrm{NC}$ & $21,20 \pm 0,01$ \\
\hline **Jun/12 & NC & NC & $24,40 \pm 0,01$ & NC & $21,25 \pm 0,02$ \\
\hline $\mathrm{Cu}$ & P2 & P3 & $\mathrm{P} 4$ & P2 & P3 \\
\hline *Out/11 & NC & $5,40 \pm 2,01$ & $5,30 \pm 0,02$ & $5,00 \pm 0,02$ & NC \\
\hline${ }^{*}$ Dez/11 & NC & NC & $\mathrm{NC}$ & $5,00 \pm 0,01$ & $4,80 \pm 0,01$ \\
\hline${ }^{*} \mathrm{Fev} / 12$ & $5,50 \pm 0,01$ & $5,60 \pm 0,01$ & $5,50 \pm 0,01$ & NC & $4,90 \pm 0,02$ \\
\hline${ }^{* *} \mathrm{Abr} / 12$ & $6,05 \pm 0,01$ & NC & NC & NC & $4,90 \pm 0,01$ \\
\hline${ }^{* *} \mathrm{Jun} / 12$ & $\mathrm{NC}$ & NC & $6,80 \pm 0,13$ & NC & $4,95 \pm 0,01$ \\
\hline $\mathrm{Fe}$ & $\mathrm{P} 2$ & P3 & $\mathrm{P} 4$ & P2 & P3 \\
\hline *Out/11 & NC & $42,60 \pm 0,02$ & $42,80 \pm 0,04$ & $39,15 \pm 0,01$ & $\mathrm{NC}$ \\
\hline${ }^{*}$ Dez/11 & NC & $\mathrm{NC}$ & NC & $39,09 \pm 0,01$ & $39,60 \pm 0,02$ \\
\hline${ }^{*} \mathrm{Fev} / 12$ & $42,90 \pm 0,01$ & $42,80 \pm 0,01$ & $42,95 \pm 0,01$ & $40,30 \pm 0,01$ & $40,10 \pm 0,02$ \\
\hline${ }^{* *} \mathrm{Abr} / 12$ & $41,75 \pm 0,01$ & $\mathrm{NC}$ & $\mathrm{NC}$ & NC & $40,50 \pm 0,02$ \\
\hline${ }^{* *} \mathrm{Jun} / 12$ & $\mathrm{NC}$ & NC & $41,60 \pm 0,01$ & NC & $40,40 \pm 0,01$ \\
\hline \multicolumn{3}{|c|}{ Leporinus friderici } & \multicolumn{3}{|c|}{ Hypostomus strigaticeps } \\
\hline $\mathrm{Zn}$ & \multicolumn{2}{|c|}{$\mathrm{P} 4$} & P2 & P3 & $\mathrm{P} 4$ \\
\hline${ }^{*}$ Out/11 & \multicolumn{2}{|c|}{$20,30 \pm 0,01$} & $26,90 \pm 0,02$ & $\mathrm{NC}$ & NC \\
\hline${ }^{*}$ Dez/11 & \multicolumn{2}{|c|}{$21,40 \pm 0,01$} & $\mathrm{NC}$ & $\mathrm{NC}$ & NC \\
\hline${ }^{*}$ Fev/12 & \multicolumn{2}{|c|}{$\mathrm{NC}$} & NC & $27,25 \pm 0,01$ & $27,00 \pm 0,01$ \\
\hline${ }^{* *} \mathrm{Abr} / 12$ & \multicolumn{2}{|c|}{ NC } & $27,40 \pm 0,02$ & $\mathrm{NC}$ & NC \\
\hline **Jun/12 & \multicolumn{2}{|c|}{ NC } & $\mathrm{NC}$ & NC & $28,10 \pm 0,02$ \\
\hline $\mathrm{Cu}$ & \multicolumn{2}{|c|}{ P4 } & P2 & P3 & P4 \\
\hline${ }^{*}$ Out/11 & \multicolumn{2}{|c|}{$5,10 \pm 0,03$} & $6,00 \pm 0,02$ & NC & NC \\
\hline${ }^{*}$ Dez/11 & \multicolumn{2}{|c|}{$4,90 \pm 0,01$} & $\mathrm{NC}$ & NC & $\mathrm{NC}$ \\
\hline${ }^{*} \mathrm{Fev} / 12$ & \multicolumn{2}{|c|}{$4,90 \pm 0,01$} & $\mathrm{NC}$ & $7,15 \pm 0,01$ & $7,00 \pm 0,01$ \\
\hline${ }^{* *} \mathrm{Abr} / 12$ & \multicolumn{2}{|c|}{$\mathrm{NC}$} & $7,60 \pm 0,02$ & $\mathrm{NC}$ & NC \\
\hline${ }^{* *} \mathrm{Jun} / 12$ & \multicolumn{2}{|c|}{$\mathrm{NC}$} & $\mathrm{NC}$ & $\mathrm{NC}$ & $7,80 \pm 0,03$ \\
\hline $\mathrm{Fe}$ & \multicolumn{2}{|c|}{$\mathrm{P} 4$} & P2 & P3 & $\mathrm{P} 4$ \\
\hline${ }^{*}$ Out/11 & \multicolumn{2}{|c|}{$38,90 \pm 0,02$} & $45,60 \pm 0,03$ & NC & $\mathrm{NC}$ \\
\hline${ }^{*}$ Dez/11 & \multicolumn{2}{|c|}{$40,20 \pm 0,01$} & $\mathrm{NC}$ & NC & $\mathrm{NC}$ \\
\hline${ }^{*}$ Fev/12 & \multicolumn{2}{|c|}{ NC } & $\mathrm{NC}$ & $50,75 \pm 0,01$ & $50,20 \pm 0,01$ \\
\hline${ }^{* *} \mathrm{Abr} / 12$ & \multicolumn{2}{|c|}{ NC } & $49,80 \pm 0,01$ & $\mathrm{NC}$ & $\mathrm{NC}$ \\
\hline${ }^{* *} \mathrm{Jun} / 12$ & \multicolumn{2}{|c|}{$\mathrm{NC}$} & $\mathrm{NC}$ & $\mathrm{NC}$ & $49,75 \pm 0,11$ \\
\hline
\end{tabular}

NC: não coletado. ANVISA 685/1998b valores máximos permitidos no pescado in natura: Zn: $50 \mathrm{mg} / \mathrm{Kg}$; Cu: $30 \mathrm{mg} / \mathrm{Kg}$; Fe: valor não estipulado. ANVISA 33/1998a Ingestão Diária Recomendada (IDR) para um adulto: Zn: 15 mg; Cu: 3 mg; Fe: 14 mg. *Meses que correspondem à estação chuvosa, **Meses que correspondem à estação seca.

Segundo Dural et al. (2007), o acúmulo de metais ocorre em maior quantidade no fígado e nas brânquias e, em menor quantidade, na musculatura dos peixes, sendo os primeiros, considerados bons bioindicadores da exposição por metais por serem responsáveis por metabolizá-los. Entretanto, a musculatura é a parte que, em geral, é consumida pela população, assim, o conhecimento da concentração de metais pesados em peixes é 
importante, no que diz respeito à gestão do meio ambiente e à saúde humana.

Nas três espécies de peixes analisadas, evidenciaram-se pequenas variações nas proporções de $\mathrm{Zn}, \mathrm{Cu}$ e $\mathrm{Fe}$, exceto para $A$. altiparanae e $H$. strigaticeps na coleta de dezembro de 2011, quando as espécies não apareceram na amostragem.

Os valores médios de $\mathrm{Zn}$ variaram entre $22,20-28,10 \mathrm{mg} / \mathrm{kg}$ e os de $\mathrm{Cu}$, entre 4,90-7,80 $\mathrm{mg} / \mathrm{kg}$; ambos não ultrapassaram valores permitidos pela ANVISA, Portaria $n^{\circ} 685$ de 27 de agosto de 1998b, para pescado in natura, sendo de $50 \mathrm{mg} / \mathrm{kg}$ e $30 \mathrm{mg} / \mathrm{kg}$, respectivamente. O Fe apresentou valores médios entre 39,00-50,40 $\mathrm{mg} / \mathrm{kg}$, porém, não há valores máximos estipulados para este metal (Tabela 3).

Em termos de nutrição humana, observou-se que os peixes avaliados são boas fontes desses metais, já que, se um indivíduo adulto consumir uma porção de peixe de $200 \mathrm{~g}$, estará ingerindo em média entre $30-37 \%$ de $\mathrm{Zn}, 33-52 \%$ de $\mathrm{Cu}$ e $56-72 \%$ de $\mathrm{Fe}$, considerando a relação dos valores de ingestão diária recomendada (IDR) para um indivíduo adulto pela Portaria ANVISA $\mathrm{n}^{\circ} 33$ de 13 de janeiro de 1998a (Tabela 3).

De acordo com os resultados obtidos, foi possível observar que a maior variação nas concentrações dos metais nos períodos avaliados ocorreu para A. altiparanae, em que as concentrações de $\mathrm{Zn}$ e $\mathrm{Cu}$ aumentaram de um período para outro e, a concentração de $\mathrm{Fe}$, diminuiu (Tabela 3). Alguns trabalhos descritos na literatura demonstraram que vários fatores podem influenciar no acúmulo de metais em tecidos de peixes, dentre eles, a estação de coleta e os parâmetros físico-químicos da água (Kargin, 1996; Jezierska e Witeska, 2001).

Em L. friderici, as concentrações de $\mathrm{Zn}, \mathrm{Cu}$ e Fe permaneceram constantes nos dois períodos de coleta (Tabela 3). Por outro lado, em $H$. strigaticeps, apenas as concentrações de $\mathrm{Zn}$ sofreram alterações, apresentando um aumento nas concentrações (Tabela 3). Zn foi o metal que mais evidenciou a diferença entre os pontos, mesmo não sendo o que apareceu em maior concentração, entretanto, foi o metal que apresentou variações nas espécies $A$. altiparanae e $H$. strigaticeps, sendo este o fato que o destaca dos demais metais (Tabela 3)

Quanto às concentrações de $\mathrm{Zn}$, Fe e $\mathrm{Cu}$, evidenciou-se que foram maiores em $H$. strigaticeps (peixe de fundo) e menores em $L$. friderici (peixe de superfície). Em relação aos pontos de coleta, as maiores concentrações foram obtidas nos pontos 3 e 4; fato que pode ser atribuído à maior movimentação ou lixiviação nos mesmos, uma vez que se tratam de locais próximos à foz do rio e nas proximidades de uma estação de recolhimento de embalagens de agrotóxicos (Figura 1).

Quanto aos níveis consideráveis de $\mathrm{Zn}$, Birungi et al. (2007) mencionam que práticas agrícolas com aplicações de insumos podem contribuir para a lixiviação de alguns metais para vias aquáticas; os autores mencionam também que as acentuadas concentrações de Fe devem-se, principalmente, ao fato de o metal estar presente naturalmente no meio ambiente.

Rahman et al. (2012) estudaram as concentrações de metais pesados nos músculos de 10 espécies de peixes coletados em duas estações diferentes no rio Bangshi em Bangladesh e evidenciaram valores elevados de Zn (entre 42,83- 418,05 mg/kg), porém, não acima dos valores permitidos na região de coleta; além disso, determinaram valores de $\mathrm{Cu}$ entre 8,33$43,18 \mathrm{mg} / \mathrm{kg}$ em todas as amostras, ambos valores significativamente elevados comparados com os dados apresentados na Tabela 3. Segundo Lima Júnior et al. (2002), baixas concentrações de $\mathrm{Cu}$ são normais em peixes e, para detectar as maiores concentrações, é necessário um estudo detalhado das vísceras.

Mendil et al. (2005) determinaram concentrações traço em diversas espécies de peixes, em que as concentrações de metais decresceram na ordem: $\mathrm{Fe}, \mathrm{Zn}, \mathrm{Cu}, \mathrm{Ni}$ e $\mathrm{Cr}$. As concentrações de Fe apresentaram-se com valor máximo de 197,00 $\mu \mathrm{g} / \mathrm{g}$ para a espécie Capoetacapoeta e valor mínimo de $64,30 \mu \mathrm{g} / \mathrm{g}$ para a espécie Leiciscuscephalus e, segundo os autores, estes valores são mais elevados do que os já descritos na literatura. Quanto ao $\mathrm{Zn}$, as concentrações variaram entre $38,60 \mu \mathrm{g} / \mathrm{g}$ em Engrauliusencrasicholus e $11,90 \mu \mathrm{\mu g} / \mathrm{g}$ em Capoetatincae; já as concentrações de Cu variaram entre 4,10 e 1,00 $\mu \mathrm{g} / \mathrm{g}$ em todas as espécies. Estes resultados são semelhantes aos determinados neste estudo.

Em outro estudo, Mendil et al. (2010), avaliando cinco espécies de peixes em um rio da Turquia, evidenciaram, nas amostras, $\mathrm{Fe}$ em maior concentração, seguido de $\mathrm{Zn}, \mathrm{Cu}$ e $\mathrm{Ni}$, 
dentre outros metais analisados. Os autores citam que as maiores concentrações são encontradas na temporada de verão e que ocorre um alto acúmulo de Fe e $\mathrm{Zn}$ em Leiciscuscephalus e $\mathrm{Cu}$ e $\mathrm{Ni}$ em Chondrostomareguim. Em nosso estudo, a estação de seca, caracterizada como outono e inverno, foram as que apresentaram os valores destes metais em maior concentração em duas (A. altiparanae e $H$. strigaticeps) das três espécies analisadas.

Gorur et al. (2012) determinaram níveis de metais pesados em quatro espécies de peixes comuns na Turquia (Engraulisencrasicholus, Oncorhynchusmykiss, Trachurusmediterranus e Merlagiusmerlangus), e observaram concentrações de Fe variando entre 7,91 e 16,06 $\mu \mathrm{g} / \mathrm{g}$ para as espécies, sendo estes, valores menores dos detectados neste estudo, nas três espécies. Os níveis de $\mathrm{Zn}$ apresentados pelos autores variaram entre 7,48 e $11,67 \mu \mathrm{g} / \mathrm{g}$ para $O$. mykiss, entre 13,03 e 17,09 $\mu \mathrm{g} / \mathrm{g}$ para E. encrasicholus, entre 5,11 e 9,63 $\mu \mathrm{g} / \mathrm{g}$ para T. mediterranus e entre 4,69 e 15,34 $\mu \mathrm{g} / \mathrm{g}$ para $M$. merlangus, sendo todos os valores bastante baixos quando comparados aos deste estudo. As concentrações de $\mathrm{Cu}$ foram de $0,40 \mu \mathrm{g} / \mathrm{g}$ para T. mediterranus e $1,21 \mu \mathrm{g} / \mathrm{g}$ para O. mykis, também abaixo dos valores determinados neste estudo.

Quanto aos metais $\mathrm{Cr}$ e $\mathrm{Ni}$, estes não foram detectados em nenhuma das amostras de peixe deste estudo e, segundo Martins (2004), Cr é raramente encontrado em águas naturais e quando isto ocorre, os efeitos sobre peixes são brandos, pois estes suportam bem a presença deste metal, diferente do que ocorre com outras formas de vida aquáticas. Já o $\mathrm{Ni}$, segundo Furtado (2007), tem seu nível tóxico dependente dos valores de $\mathrm{pH}$, sendo considerados mais tóxicos às plantas aquáticas do que aos peixes propriamente ditos.

Honda et al. (1979) citam que os metais podem ser encontrados mais facilmente no tecido da pele dos peixes do que dos músculos, sendo proporcional ao grau de pigmentação, uma vez que cromatóforos e eridócitos são bem distribuídos nesses tecidos, e os autores acreditam que a concentração de Zn seja maior em peixes mais escuros, o que condiz com este estudo, em que evidenciou-se que as maiores concentrações de $\mathrm{Zn}$ foram determinadas em $H$. strigaticeps, que é o peixe de coloração mais escura
Sing et al. (1991), ao analisar as concentrações de metais pesados em cinco espécies de peixes, constatou que $\mathrm{Zn}$, $\mathrm{Cu}$ e $\mathrm{Fe}$, em algumas das amostras, variaram conforme o tamanho do espécime e, em sua maioria, a concentração aumentou conforme aumentou o tamanho. Os autores citam que deve-se considerar as taxas de excreção de cada espécie, que com o tempo podem reduzir a concentração de metais nos organismos e que o acúmulo de metal a nível intra específico seja reforçado com o tempo de exposição. Estes fatores foram observados neste estudo, uma vez que o peixe de menor tamanho (A. altiparanae) foi o que apresentou as menores concentrações dos metais.

Estudos como este que avaliam metais em peixes são importantes, devido ao fato de que os peixes do córrego contribuem para o equilíbrio global do ambiente e também são consumidos pela população. $\mathrm{O}$ consumo de peixes é extremamente importante para a saúde devido à sua fonte de proteína, ácidos graxos e metais (Copat et al., 2012).

\section{Conclusões}

O estudo possibilitou avaliar a presença de metais em peixes no córrego Curral de Arame no Município de Dourados/MS.

Os resultados apresentados indicam que as amostras de peixes não excederam as concentrações de metais permitidas pela ANVISA, Portaria 685/1998b.

Os peixes avaliados mostraram-se boas fontes dos metais analisados, considerando os valores de Ingestão Diária Recomendada (IDR) pela ANVISA na Portaria 33/1998a.

$\mathrm{O}$ peixe de fundo apresentou maior concentração de metais, em geral, em relação às outras espécies, e os resultados sofreram a influência dos pontos de coleta.

\section{Agradecimentos}

Agência Brasileira de Inovação (FINEP), Coordenação Aperfeiçoamento de Pessoal de Nível Superior (CAPES), Fundação de Apoio ao Desenvolvimento do Ensino, Ciência e Tecnologia do Estado de Mato Grosso do Sul (FUNDECT) e Conselho Nacional de Desenvolvimento Científico e Tecnológico (CNPq). 


\section{Referências}

ANVISA - Agência Nacional de Vigilância Sanitária, Portaria $\mathrm{n}^{\circ} 33$ de 13 de janeiro de 1998a. Disponível em: $<$ http://www.anvisa.gov.br/legis/portarias/33 98.htm>. Acessado em: 20 de jan. de 2013.

ANVISA, Agência Nacional de Vigilância Sanitária, Portaria $\mathrm{n}^{\circ} 685$ de 27 de agosto de 1998b. Disponível em: $<$ http://www.anvisa.gov.br/legis/portarias/68 5 98.htm>. Acessado em: 03 de out. de 2012.

\section{BENEDITO-CECILIO E, PEREIRA AL,} BALERONI H, FARIA ACEA 2005. Effects of habitat on physiological indicators in Leporinus friderici (Pieces, Anostomidae) in the influence area of the Corumbá reservoir, Goiás, Brazil. Acta Liminologica Brasiliensis 17: 71-79.

BIRUNGI Z, MAZELA B, ZARANYIKA MF, NAIGAGA I, MARSHAL B 2007. Active biomonitoring of trace heavy metals using fish (Oreochromis niloticus) as bioindicator species. The case of Nakivubo wetland along Lake Victoria. Physics and Chemistry of the Earth 32:1350-1358.

BRAGA FMS 2001. Crescimento e mortalidade de Leporinus friderici (Ostariophysi, Anostomidae) na represa de Volta Grande, rio Grande, localizada entre os estados de Minas Gerais e São Paulo, Brasil. Acta Scientarum 23:415-420.

CLESCERI LS, EATON AD, GREENBERG AE, FRASON MA 1996. Standard methods for the examination of water and wasterwater. 19 th ed. Supplement. Washington, DC.

DURAL M, GOKSU MZL, OZAK AA 2007. Investigation of heavy metals levels in economically important fish species captured from the Tuzla lagoon. Food Chemistry 102:415-421.

FURTADO JGC 2007. Estudos de Impactos Ambientais causados por metais pesados em água do mar na Baía de São Marcos: Correlações e níveis Background. Brasil, ?p. Dissertação (Mestrado em Química) Universidade Federal da Paraíba, Paraíba.

GOMIERO LM, BRAGA FMS 2003. O lambari Astyanax altiparanae (Characidae) pode ser um dispersor de sementes?. Acta Scientiarum Biological Sciences 25:353-360.

GORUR FK, KESER R, AKÇAY N, DIZMAN S 2012. Radioactivity and heavy metals concentration of some commercial fish species consumed in the Black Sea Region of Turkey. Chemosphere 87:356-361.

HONDA K, MATSUDA M, TATSUKAWA R 1979. Distribution of heavy metals and its characteristic in albacore (Thunnus alalunda) and bonito (Katsuwonus pelamis). Nippon Kagaku Kaishi 53:177-182.

JEZIERSKA B, WITESKA M 2001. Metal toxicity to fish. Wydawnictwo Akademii Podlaskiej, Siedlce.

KALAY M, CANLI M 2000. Elimination of essential $(\mathrm{Cu}, \mathrm{Zn})$ metals from tissues of a freshwater fish Tilapia Zilli. Journal of Zoology 24:429-436.

KARGIN F 1996. Water Seasonal changes in Levels of Heavy Metals in tissues of Mullus barbatus and Sparus aurata collected from Iskenderun Gulf (Turkey). Air and Soil Pollution 90:557-562.

LEMES MJL 2001. Avaliação de metais e elementos traço em águas e sedimentos das bacias hidrográficas dos rios Mogi Guaçu e 
Pardo. Dissertação. (Mestrado em Ciências) Instituto de Pesquisas Energéticas e Nucleares (IPEN), São Paulo.

LIMA JÚNIOR RG, ARAÚJO FG, MAIA MF, PINTO ASSB 2002. Evaluation of Heavy Metals in fish of the Sepetiba and Ilha Grande Bays, Rio de Janeiro, Brazil. Environment Research 89:171-179.

MARTINS RJE 2004. Acumulação e libertação de metais pesados por briófitas aquáticas. Tese (Doutorado em Engenharia Química) Universidade do Porto, Portugal.

MENDIL D, ULUOZLU OD, HASDEMIR E, TUZEN M, SARI H, SUIÇMEZ M 2005. Determination of trace metal levels in seven fish species in lakes in Tokat, Turkey. Food Chemistry 90:175-179.

MENDIL D, UNAL OF, TUZEN M, SOLYLAK M 2010. Determination of trace metals in different fish species and sediments from the River Yesilirmak in Tokat, Turkey. Food and Chemical Toxicology 48:1383-1392.

ORSI ML, CARVALHO ED, FORESTI F 2004. Biologia populacional de Astyanax altiparanae Garutti\&Britski (Teleostei, Characidae) do médio Rio Paranapanema, Paraná, Brasil. Revista Brasileira de Zoologia 21:207-218.

RAHMAN MS, MOLLA AH, SAHA N, RAHMAN A 2012. Study on heavy metals levels and its risk assesment in some edible fishes from Bangshi River, Savar, Dhaka, Bangladesh. Food Chemistry 134:1847-1854.

ROSSO GT 2013. Avaliação de metais e pesticidas em peixes, águas e sedimentos coletados no Córrego Curral de Arame, em Dourados, MS. Dissertação (Mestrado em Recursos naturais). Universidade Estadual de Mato Grosso do Sul
SING JG, CHANG-YEN I, STOUTI VA, CHATERGOON L 1991. Distribution of selected heavy metals in skin and muscle of five tropical marine fishes. Environmental Pollution 69:302-215.

UNEP - United Nations Environment Program. UNEP 2010 Annual Report. 2011. Disponível em:

$<$ http://www.unep.org/publications/contents /pub_details_search.asp?ID=4186> Acessado em: 12 de dez. de 2013.

VANDECASTEELE C, BLOCK B 1991. Modern methods for trace element determination. $1^{\underline{a}}$ ed., New York, John Wiley \& Sons.

VIRGA RHP, GERALDO LP, SANTOS FH 2007. Avaliação da contaminação por metais pesados em amostras de siris azuis. Ciência e Tecnologia de Alimentos 27:779-785. 